\title{
Zur interaktiven Aushandlung von Teilnehmerkategorien in interkultureller Kommunikation
}

\author{
Birte Asmuß (Sønderborg)
}

\begin{abstract}
In intercultural communication, the training of intercultural competence is seen as one of the primary solutions to problems and misunderstandings arising in intercultural situations. Intercultural competence is based on the assumption that specific cultural based factors have a continuous influence on the communication.

In this article it is argued for that this assumption can be a problematic one. Based on an analysis of authentic talk-in-interaction between two interlocutors from different nations it is shown that interlocutors continuously negotiate about membership categories like e.g. cultural origin and its local relevance for the talk. This means that cultural origin is not a continuous, but a dynamic entity. This leads to the suggestion that in intercultural communication the training of interactive strategies should be an alternative or a necessary addition to traditional training aims like intercultural competence.
\end{abstract}

\section{$1 \quad$ Einführung}

Dieser Artikel stellt einen Beitrag zur Diskussion um interkulturelle Kommunikation und damit verbunden dem Begriff der interkulturellen Kompetenz dar. Ausgehend von einer Analyse authentischer Interaktion zwischen Vertretern zweier unterschiedlicher Nationen wird diskutiert, welche Bedeutung die wiederkehrende interaktive Aushandlung von Teilnehmerkategorien im Gespräch für die Diskussion um den Gegenstand interkulturelle Kommunikation und das Lernziel interkulturelle Kompetenz hat.

\section{Interkulturelle Kommunikation und interkulturelle Kompetenz}

Im Forschungsbereich interkulturelle Kommunikation ${ }^{1}$ gilt die Vermittlung interkultureller Kompetenz nahezu unbestritten als Garant für das Gelingen der Kommunikation zwischen

\footnotetext{
${ }^{1}$ Unter dem Begriff 'interkulturelle Kommunikation' wird Unterschiedliches verstanden. Ich werde mich einer Diskussion hierüber im Rahmen dieses Artikels nicht stellen. Stattdessen muss hier der Hinweis genügen, dass der Begriff interkulturelle Kommunikation in dieser Untersuchung dafür steht, dass Kommunikation zwischen Vertretern unterschiedlicher Nationen stattfindet, die eine unterschiedliche Muttersprache sprechen. Dies geschieht aus der Überlegung heraus, dass Nationen ebenso wie Sprachgebiete Entitäten darstellen können, an die eine kulturelle Zugehörigkeit gebunden ist. Zur Diskussion um den Begriff 'interkulturelle Kommunikation' s.a. Hinnenkamp 1994, Knapp/Knapp-Potthoff 1990, Knapp-Potthoff 1997, Krumm 1995, Rost-Roth 1994.
} 
Vertretern unterschiedlicher kultureller Zugehörigkeit. Umstrittener ist jedoch die Frage, was unter interkultureller Kompetenz eigentlich zu verstehen ist. Hier lassen sich (mindestens) zwei grundlegende Haltungen erkennen. Zum einen diejenige, die unter interkultureller Kompetenz die Ausstattung der Lernenden mit einem Wissen über die andere Kultur versteht. Hier steht die Vermittlung von Fakten über die andere Kultur und die landesspezifischen Gegebenheiten sowohl sprachlich, kulturell als auch gesellschaftlich, politisch und geographisch im Zentrum. Zum anderen diejenige, die eine Sensibilisierung gegenüber dem Fremden als zentrales Element ansieht. Hierbei geht es weniger um die Ausstattung der Lernenden mit einem faktuellen Wissen über landes- und kulturspezifische Gegebenheiten als über die Initiierung und Etablierung eines Bewusstwerdungsprozesses über das Fremde an sich und über bestimmte Reaktionen auf die Begegnung mit dem Unbekannten.

Während der erste Ansatz primär die Vermittlung interkultureller Kompetenz in Bezug auf eine bestimmte Zielkultur anstrebt, versucht der zweite in stärkerem Maße eine allgemein gültige interkulturelle Kompetenz zu vermitteln, die in Bezug auf eine Vielzahl unterschiedliche Kulturen einsetzbar ist. Der erste Ansatz hat sich primär in stark anwendungsorientierten Bereichen wie Trainingsprogrammen zur interkulturellen Kommunikation durchgesetzt (Andersen 1997, Bergemann 1996, Opitz 1997). Der zweite findet sich primär in Lehrwerken zur Fremdsprachenvermittlung, in denen dem Aspekt der Kultur ein großer Stellenwert eingeräumt wird (z.B. Bachmann et al. 1995).

So unterschiedlich diese Ansätze in Bezug auf Zielsetzung und Zielkultur auch sein mögen, so sehr stimmen sie in ihrer grundlegenden Haltung überein, dass die Ursachen für Probleme in interkultureller Kommunikation auf Unterschieden zwischen den Kulturen beruhen. Hiermit wird die unterschiedliche kulturelle Zugehörigkeit der Gesprächsteilnehmer in interkulturellen Kommunikationssituationen als Ursache für entstehende offene oder auch verdeckte Missverständnisse und Probleme angesehen. Die Entstehung dieser Probleme und Missverständnisse kann den skizzierten Ansätzen zufolge dadurch vermieden werden, dass ein Wissen über die und eine Sensibilisierung gegenüber der Fremdkultur vermittelt wird.

Durch die Fokussierung auf Unterschiede wird demnach die kulturelle und damit oft einhergehend nationale Zugehörigkeit der Gesprächsteilnehmer als der Faktor angesehen, der die Interaktion bestimmt. Doch in diesem Zusammenhang stellt sich die Frage, ob die nationale oder kulturelle Zugehörigkeit der allzeit entscheidende Faktor in der Interaktion ist. Spielt die nationale oder kulturelle Zugehörigkeit in jeder Gesprächssituation und in jeder Phase des Gesprächs die gleiche Rolle?

Eine Möglichkeit dies für eine Form interkultureller Kommunikation, nämlich die mündliche, zu überprüfen, ist zu untersuchen, wie Gesprächsteilnehmer einander im Gespräch bestimmte Aspekte des Kontextes wie beispielsweise die Kultur zuweisen und einander bestimmte nationale Zugehörigkeiten im Gespräch relevant machen. 


\section{Teilnehmerkategorien}

Studien zu Teilnehmerkategorien ('membership categories', Sacks 1992) machen deutlich, wie Teilnehmerkategorien in Gesprächen interaktiv, d.h. von den Gesprächsteilnehmern gemeinsam, ausgehandelt werden (z.B. Hester/Eglin 1997, Kesselheim 1998). In diesen Studien wird deutlich, dass Größen wie Kontext und hierunter auch der Aspekt der kulturellen Zugehörigkeit gesprächsinterne Faktoren sind, über deren Relevanz lokal im Gespräch verhandelt wird. Studien dieser Art stellen damit Ansätze in Frage, in denen Kontext als gesprächsexterne Größe als bedeutend für das Gespräch betrachtet wird (z.B. Andersen 1997, Fredsted 1998, Koch/Rossenbeck 1997).

Sacks (1992) schreibt zur Bedeutung von Teilnehmerkategorien und zur interaktiven Zuschreibung derselben:

It's perfectly obvious that there is a range of sets whose categories could be used; from the set 'sex', "a woman". From 'race', "a Negro". From 'religion', "a Catholic". From 'occupation', "a psychiatric social worker", etc., etc. Each of these categories could apply to the same person. And it's perfectly obvious that Members do use one set's categories for some statements and another set's categories for other statements. (Sacks 1992, Lecture 6, S. 41)

Hiermit hebt Sacks hervor, dass für ein und dieselbe Person unterschiedliche Kategorien relevant sein können: von der Kategorie Geschlecht beispielsweise 'Frau', von der Religion 'Katholik', vom Beruf 'Sozialarbeiter'. Welche dieser unterschiedlichen Kategorien in welcher Phase eines Gesprächs entscheidend ist, ist nicht textextern im Vorhinein zu entscheiden, sondern wird textintern von den Gesprächsteilnehmern im Gespräch selbst ausgehandelt. Dies bedeutet auch, dass nicht einer bestimmten Kategorie während eines gesamten Gesprächs eine gleichbleibende Bedeutung zugeschrieben werden kann. Stattdessen werden im Gespräch lokal immer wieder unterschiedliche Kategorien durch die Gesprächsteilnehmer als relevant ausgehandelt. Insofern sind Teilnehmerkategorien dynamische Konstrukte, deren Bedeutung für die Gesprächsteilnehmer im Gespräch selbst kontinuierlich ausgehandelt wird.

An Sacks Überlegungen schließt sich die Frage an, inwieweit es bestimmte Prozeduren gibt, nach denen Gesprächsteilnehmer bestimmte Kategorien auswählen und sie einander zuweisen (Sacks 1992, Lecture 6, S. 42). Eine Aufgabe der Analyse von Teilnehmerkategorien besteht demnach darin zu untersuchen, mit welchen Mitteln eine solche interaktive Aushandlung von Teilnehmerkategorien im Gespräch stattfindet, welche Regularitäten in diesem Zusammenhang auftreten und inwiefern eine Orientierung der Gesprächsteilnehmer an diesen Regularitäten nachzuweisen ist.

In diesem Zusammenhang ist die Rolle des Kontextes, in Bezug auf interkulturelle Kommunikation insbesondere der Aspekt der Kultur, von entscheidender Bedeutung. Hier weisen Studien zu Kontextualisierungshinweisen interessante Ergebnisse auf, die im Folgenden kurz skizziert werden sollen. 


\section{Kontextualisierungshinweise}

Kontextualisierungshinweise geben den Gesprächsteilnehmern im Verlauf des Gesprächs Anhaltspunkte darüber, welche Aspekte des Kontextes in einer bestimmten Phase des Gesprächs gerade relevant sind. Gumperz spricht von 'contextualization cues' und definiert diese folgendermaßen:

Roughly speaking, a contextualization cue is any feature of linguistic form that contributes to the signalling of contextual presuppositions. (Gumperz 1982:131)

Er hebt hervor, dass Kontextualisierungshinweise aus einer Vielzahl von linguistischen Formen bestehen, die auf unterschiedliche Weise kontextuelle Präsuppositionen signalisieren. Hiermit tragen Kontextualisierungshinweise zur gegenseitigen Verständnissicherung bei, indem sie die Gesprächsteilnehmer darüber informieren, welche Aspekte des Kontextes in welcher Phase des Gesprächs relevant sind. Kontext ist demnach als eine textinterne Größe anzusehen. Schiffrin (1994:236) schließt sich diesem Kontextverständnis an, indem sie hervorhebt, dass die Relevanz eines bestimmten Kontextes immer im Text selbst zu finden ist.

Heritage (1984) fügt der Diskussion um Kontext als textinterne Größe basierend auf Sacks/ Schegloff/Jefferson (1974:699f.) die Termini 'context-shaped' und 'context-renewing' (Heritage 1984:242) hinzu. Eine Äußerung in mündlicher Interaktion wird demnach einerseits in der Interaktion selbst geformt (context-shaped), indem sie nur durch Bezug zum Kontext, insbesondere den durch die direkt vorhergehenden Äußerungen geschaffenen, verständlich wird. Andererseits wird durch jede neue Äußerung ein neuer Kontext geschaffen, in welchem die nachfolgenden Äußerungen durch die Gesprächsteilnehmer zu deuten sind. In diesem Sinne trägt jede Äußerung auch dazu bei, den Kontext zu aktualisieren (context-renewing).

Für die nachfolgenden Analysen bedeuten die obenstehenden Überlegungen, dass genauer untersucht werden soll, mit welchen Mitteln die Gesprächsteilnehmer Kontextualisierungen im Gespräch vornehmen und wie sie die interaktive Verhandlung über den relevanten Kontext gestalten.

\section{$5 \quad$ Analyse}

Konkret soll in der folgenden Analyse der Frage nachgegangen werden, an welchen Stellen im Gespräch die Teilnehmer Aspekte kultureller Zugehörigkeit relevant machen. Hierbei soll auch die Frage behandelt werden, mittels welcher Techniken die Teilnehmer einander bestimmte kulturelle Kontexte relevant machen und wie die Kontextrelevanz interaktiv ausgehandelt wird.

Zunächst zu den Daten:2 Die Daten stammen aus einer informellen, authentischen Gesprächssituation zwischen einer Gruppe von Dänen und Deutschen. Insgesamt sind ca. 8 Teilnehmer am Gespräch beteiligt, doch zu dem Zeitpunkt des Gesprächs, der im Zentrum der Analyse stehen wird, nehmen 3 Personen teil: 1 Deutscher und 2 Dänen. Die

\footnotetext{
${ }^{2}$ Ich danke den Gesprächsteilnehmern ganz herzlich für Ihre Einwilligung, dass die Daten aufgezeichnet und im wissenschaftlichen Rahmen ausgewertet werden dürfen.
} 
Gesprächsteilnehmer kennen sich aus einem universitären Rahmen, sind aber nicht näher miteinander befreundet. Der überwiegende Teil des Gesprächs findet auf Deutsch statt, in den Fällen, in denen auf Dänisch gesprochen wird, findet sich eine Wort für Wort Übersetzung ins Deutsche direkt unterhalb der entsprechenden Zeile. Zur Zeit der Aufnahme befinden sich die Gesprächsteilnehmer in einer studentischen Kneipe. Die Daten wurden mit 2 Videokameras aufgezeichnet und sind nach dem Transkriptionssystem von Jefferson (Atkinson/Heritage (eds.) 1984) transkribiert (s. Anhang).

Im Transkript wird nicht näher bezeichnet, welcher Sprecher Deutscher und welcher Däne ist. Da ein Ziel der Analyse darin besteht, die jeweiligen Kontextualisierungen und damit die kulturelle Zugehörigkeit der Sprecher und die interaktive Aushandlung hierüber aufzuzeigen, soll nicht im Vorhinein ein Aspekt des Kontextes, nämlich die nationale Zugehörigkeit der Sprecher, als relevant hervorgehoben werden. Vielmehr soll durch die Nicht-Angabe der Blick auf die Aspekte offen gehalten werden, die die Sprecher einander gegenseitig als relevant anbieten und über deren Relevanz sie miteinander verhandeln. Im Transkript wird ersichtlich, dass die Gesprächsteilnehmer in unterschiedlichem Grad die deutsche Sprache beherrschen. Daher lassen sich die Gesprächsteilnehmer allein schon aus dem Transkript in die Gruppe der Mutter- bzw. Nicht-Muttersprachler einordnen. Dies bedeutet jedoch nicht automatisch, dass sich die Gesprächsteilnehmer an dieser Tatsache als an einem Faktor orientieren, der die Kommunikation beeinflusst. Insofern kann die Unterscheidung in Mutterversus Nicht-Muttersprachler hier eine Kontextgröße sein, die der Analytiker als die Interaktion beeinflussend herausstellt. Ob sie jedoch auch eine gesprächsinterne Rolle spielt, lässt sich nur anhand der Orientierung der Gesprächsteilnehmer an unterschiedlichen Aspekten des Kontextes nachweisen. Dies soll eine der Aufgaben der nachstehenden Analyse sein.

Das untenstehende Transkript ist ein fortlaufendes Gespräch zwischen 3 Gesprächsteilnehmern. Dieser Teil des Gesprächs ist zu Analysezwecken in drei Auszüge unterteilt, die jedoch, wie aus der fortlaufenden Zeilennummerierung ersichtlich, direkt aufeinander im Gespräch folgen.

Der erste Auszug beginnt damit, dass 3 Gesprächsteilnehmer darüber sprechen, welch gute Möglichkeiten es gibt, an der dänischen Westküste zu surfen.

\section{[Auszug 1 'die see das ist das ozean']}

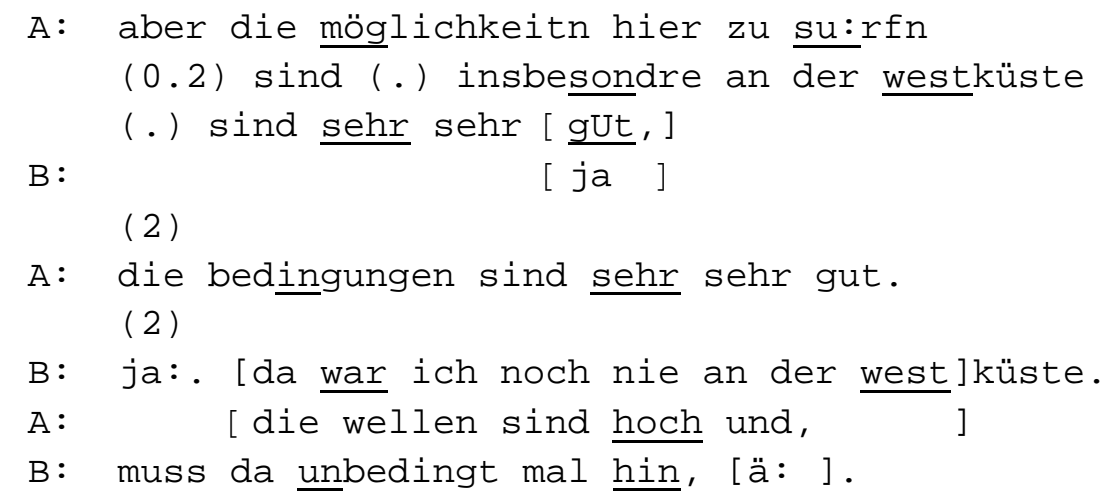




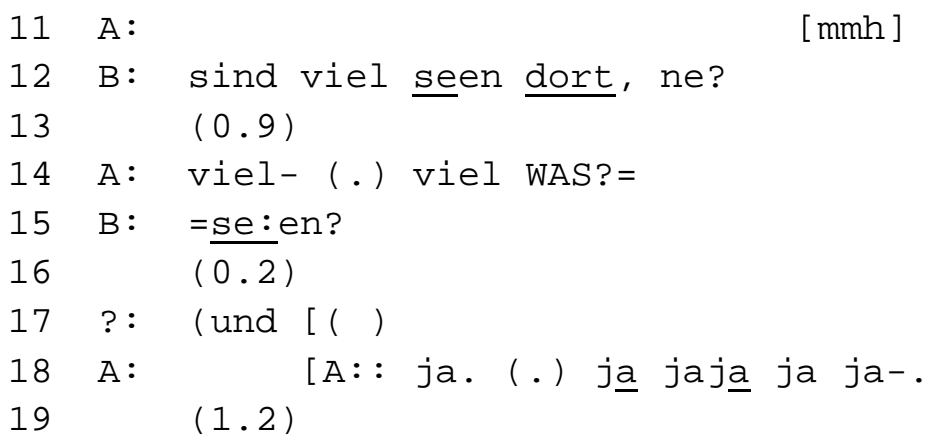

Im ersten Auszug spricht Sprecher A in Zeile 1-3 über die Möglichkeiten in Dänemark zu surfen. Sprecher B reagiert hierauf mit einem schwachen Zeichen der Akzeptanz in $4 \mathrm{ja}$. Im Anschluss hieran entsteht eine 2-sekündige Pause, die Sprecher A mit einer partiellen Wiederholung sehr sehr gut beendet. Wiederum folgt eine 2-sekündige Pause. Sprecher B übernimmt anschließend den Turn, ohne weiter auf das von Sprecher A initiierte Thema der guten Surfbedingungen einzugehen. Stattdessen relatiert Sprecher B seinen Turn zu dem geografischen Aspekt, den Sprecher A in seinem vorhergehenden Turn angeschnitten hat, nämlich der Westküste. Sprecher B markiert sich in diesem Turn als Unwissender, indem er anmerkt, dass er dort noch nie war (8). In Überschneidung mit Sprecher Bs Turn setzt Sprecher A seinen Turn fort, indem er die guten Bedingungen näher ausführt die wellen sind hoch und. Hierdurch behandelt er die Reaktion von Sprecher B auf seinen vorherigen Turn als nicht ausreichend. Sprecher B hingegen führt seinen Turn in Zeile 10 fort, ohne auf die Ausführungen von Sprecher A näher einzugehen. Stattdessen stellt er Sprecher A eine Frage, die wiederum sein Nicht-Wissen markiert und gleichzeitig Sprecher A die Rolle des Wissenden zuschreibt sind viel seen dort, ne?. Doch statt eines zweiten Teils einer Paarsequenz in Form einer Antwort durch Sprecher A entsteht eine knapp einsekündige Pause in Zeile 13. Anschließend initiiert Sprecher A eine Reparatur, indem er eine Frage stellt, die gegenseitige Verständnissicherung zum Ziel hat: viel-(.) viel WAS?. Sprecher B gibt eine Antwort in Zeile 15, die Sprecher A in Zeile 18 nach kurzem Zögern akzeptiert. Hiermit könnte die Reparatursequenz beendet sein und die Gesprächsteilnehmer könnten zum vorherigen Thema zurückkehren. Doch dies geschieht nicht, wie im Auszug 2 zu sehen sein wird.

Doch zunächst noch einmal ein Blick auf Auszug 1. Es lässt sich festhalten, dass Sprecher A und B in der ersten Phase des Gesprächs gemeinsam bestimmte Kontextualisierungen mithilfe spezifischer Kategorisierungen vornehmen. Die hier angewendeten Kontextualisierungen tragen dazu bei, Sprecher A als den Wissenden, in diesem Falle wissend in Bezug auf geografische Verhältnisse, zu konstruieren. Damit einhergehend wird Sprecher B als derjenige dargestellt, der unwissend in Bezug auf die geografischen Verhältnisse ist. Die Mittel, mit denen diese Kontextualisierungen durchgeführt werden, sind Initiierungen erster Teile von Paarsequenzen, in diesem Falle in Bezug auf Sprecher B Fragen, und responsive Handlungen in Form von zweiten Teilen von Paarsequenzen. Dies trifft auf Sprecher A zu, der Antworten auf die von Sprecher B gestellten Fragen gibt.

In dem Moment, in dem die Gesprächsteilnehmer eine Änderung der zuvor etablierten Kontextualisierungshinweise (hier in Bezug auf die Kategorien wissend vs. unwissend) 
einleiten, beginnt eine Wendung im Gespräch. Während zuvor Sprecher B noch Sprecher A die Rolle als Wissender zugeschrieben hat, werden die Rollen in der folgenden Sequenz vertauscht: Sprecher A markiert sein Verstehensproblem mit dem ersten Teil einer Paarsequenz und Sprecher B reagiert prompt mit dem angemessenen zweiten Teil in Form einer Antwort. Zu diesem Zeitpunkt könnten die Sprecher zu den zuvor relevant gemachten Kontextualisierungen zurückkehren und ihr Gespräch über die Westküste Dänemarks fortsetzen. Doch mit dem Austausch der relevanten Kontextualisierungen geht auch eine Verschiebung des inhaltlichen Fokus einher. Dies lässt sich deutlich im folgenden Auszug 2 sehen.

\section{[Auszug 2 'die see das ist das ozean']}

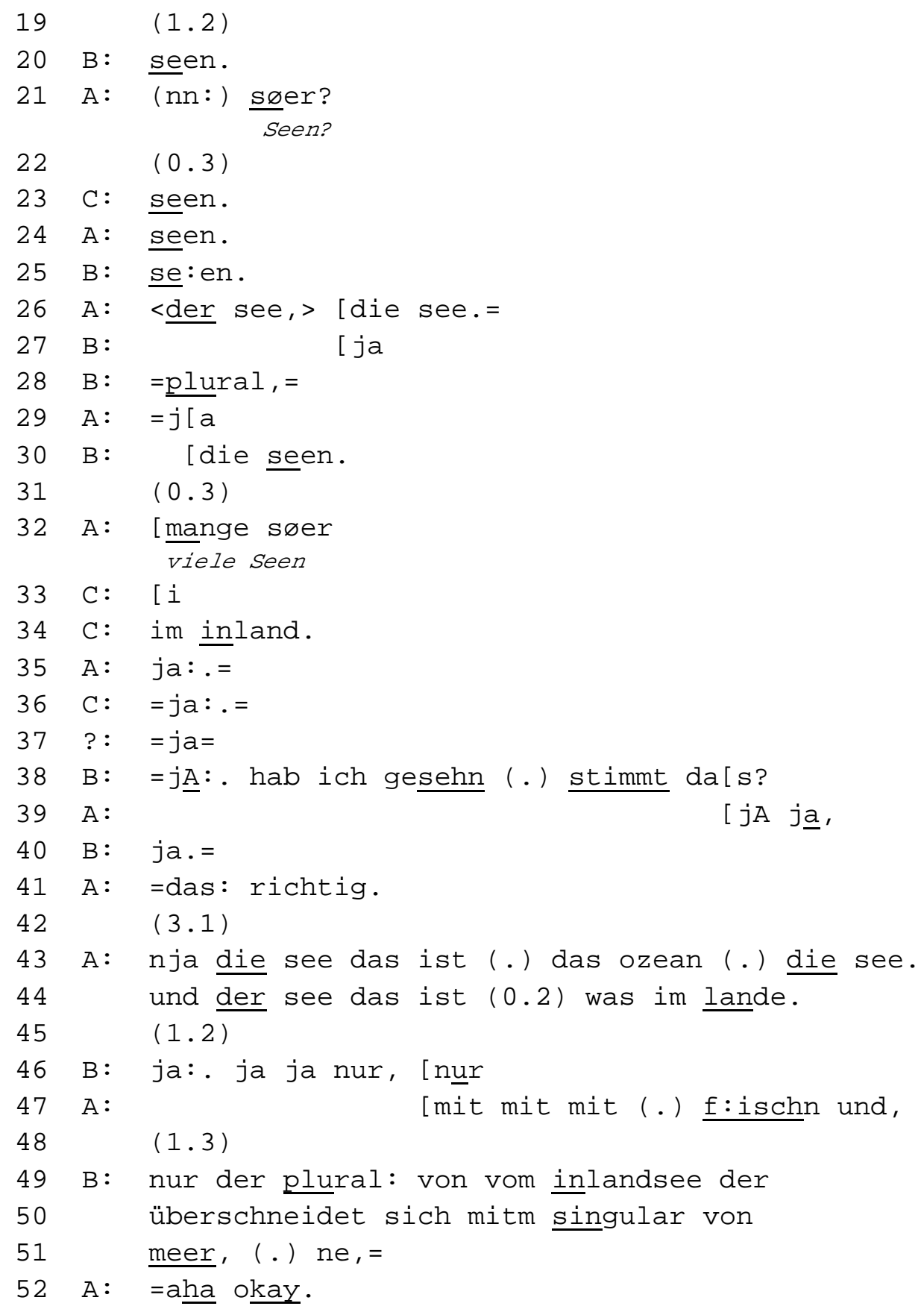




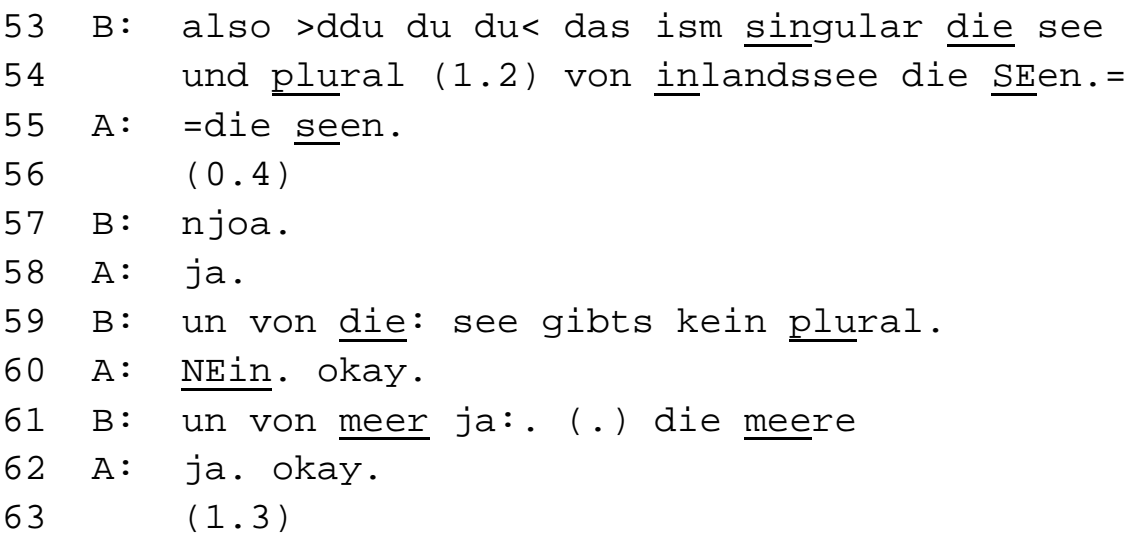

Wie schon zuvor erwähnt, besteht für die Gesprächsteilnehmer in Zeile 19 die Möglichkeit, die Reparatursequenz zu beenden und zum vorherigen Thema zurückzukehren. Doch dies geschieht nicht. Nach einer 1.2-sekündigen Pause wiederholt Sprecher B seinen zweiten Teil einer Paarsequenz seen (20), diesmal jedoch mit fallender Intonation. Hierdurch wird die zuvor eingeleitete Reparatursequenz wieder eröffnet. Ein Grund dafür, dass Sprecher B das von Sprecher A in Zeile 18 mit A:: ja markierte Verstehen nicht als ausreichend behandelt, kann darin liegen, dass Sprecher A mit der Reaktion 'ja' zwar zu erkennen gibt, dass er die von Sprecher B gegebene Antwort verstanden hat. Er zeigt aber den anderen Gesprächsteilnehmern nicht, auf welche Weise er sie verstanden hat, d.h. er ermöglicht Sprecher B keinen Einblick, ob seine Antwort 'richtig' verstanden wurde (Schegloff 1982:78). Die Wiedereröffnung der Reparatursequenz durch Sprecher B kann ein Mittel sein, um die gegenseitige Verständnissicherung zu garantieren.

Nun beginnt eine lange Verhandlung zwischen den Sprechern A, B und C darüber, was das deutsche Wort 'Seen' bedeutet. Sprecher A initiiert in Zeile 26 eine grammatische Perspektive, indem er ohne Frageintonation das Augenmerk auf Artikel und Pluralbildung von 'See' lenkt. Weiter auf die sprachliche Perspektive gerichtet sind die beiden folgenden Äußerungen zu sehen: Sprecher A übersetzt 'Seen' ins Dänische (32) und Sprecher C präzisiert in Zeile 33 und 34, dass sich 'Seen' auf Wasser im Inland und damit nicht auf das Meer bezieht. Sprecher B versucht in Zeile 38 das Thema wieder an das vor der Reparatursequenz liegende Thema anzuknüpfen. Doch in Zeile 42 wird durch die 3.1-sekündige Pause deutlich, dass das Thema von den anderen Sprechern nicht weitergeführt wird. So kehrt Sprecher A zurück zum sprachlichen Aspekt, indem er in Zeile 43 und 44 präzisiert, dass er den Unterschied zwischen 'der See' und 'die See' kennt die see das ist das ozean und der see das ist was im lande. Nun verläuft das Gespräch bis Zeile 62 über sprachliche Aspekte in Bezug auf das deutsche Wort 'See'.

Die Aspekte des Kontextes, die die Gesprächsteilnehmer einander in diesem Teil des Gesprächs relevant machen, haben zum größten Teil mit ihrem gemeinsamen Hintergrund als Sprachstudierende bzw. Lehrende zu tun. Indem sie besonders auf den sprachlichen Aspekt eingehen, treten in diesem Auszug kulturelle Zuweisungen, d.h. Kontextualisierungshinweise in Bezug auf nationale oder kulturelle Zugehörigkeit, die noch im ersten Auszug stärker im Vordergrund stehen, verstärkt in den Hintergrund. Stattdessen finden sich in einigen Äußerungsformaten Hinweise darauf, dass die Sprecher eine asymmetrische Konstellation 
untereinander in Bezug auf das aktuelle Thema 'Sprache' aufbauen. Konnte im ersten Auszug die Verteilung von Frage und Antwort als Kontextualisierunghinweise gelten, lässt sich eine solche Verteilung in Auszug 2 nicht feststellen. Während Sprecher A in Zeile 41 mit das: richtig noch markiert, dass er derjenige ist, der über genügend Wissen verfügt um beurteilen zu können, ob Sprecher Bs vorherige Feststellung richtig oder falsch ist, zeigt Sprecher A in Zeile 52, 60 und 62, dass er nun in einer anderen Rolle steckt: mit seinen Äußerungen in diesen Zeilen, die alle unterschiedliche Formen der Zustimmung darstellen, ist er nicht länger der Wissende, der Informationen austeilt, sondern derjenige, der neue (aha Zeile 52) Informationen entgegennimmt. Diese Kategorisierung weist er sich nicht alleine zu, sondern dies geschieht in enger Koordination mit Sprecher B. In dem Maße, wie Sprecher A als der Unwissende erscheint, tritt Sprecher B als der Wissende auf.

Doch diese kontextuelle Zuweisung, über die die Sprecher miteinander verhandeln und über die sie Einigkeit erzielen, bleibt im weiteren Verlauf des Gesprächs nicht bestehen. Wie im folgenden Auszug $3 \mathrm{zu}$ sehen ist, machen die Sprecher einander im weiteren Verlauf des Gesprächs erneut andere Aspekte des Kontextes relevant.

\section{[Auszug 3 'die see das ist das ozean']}

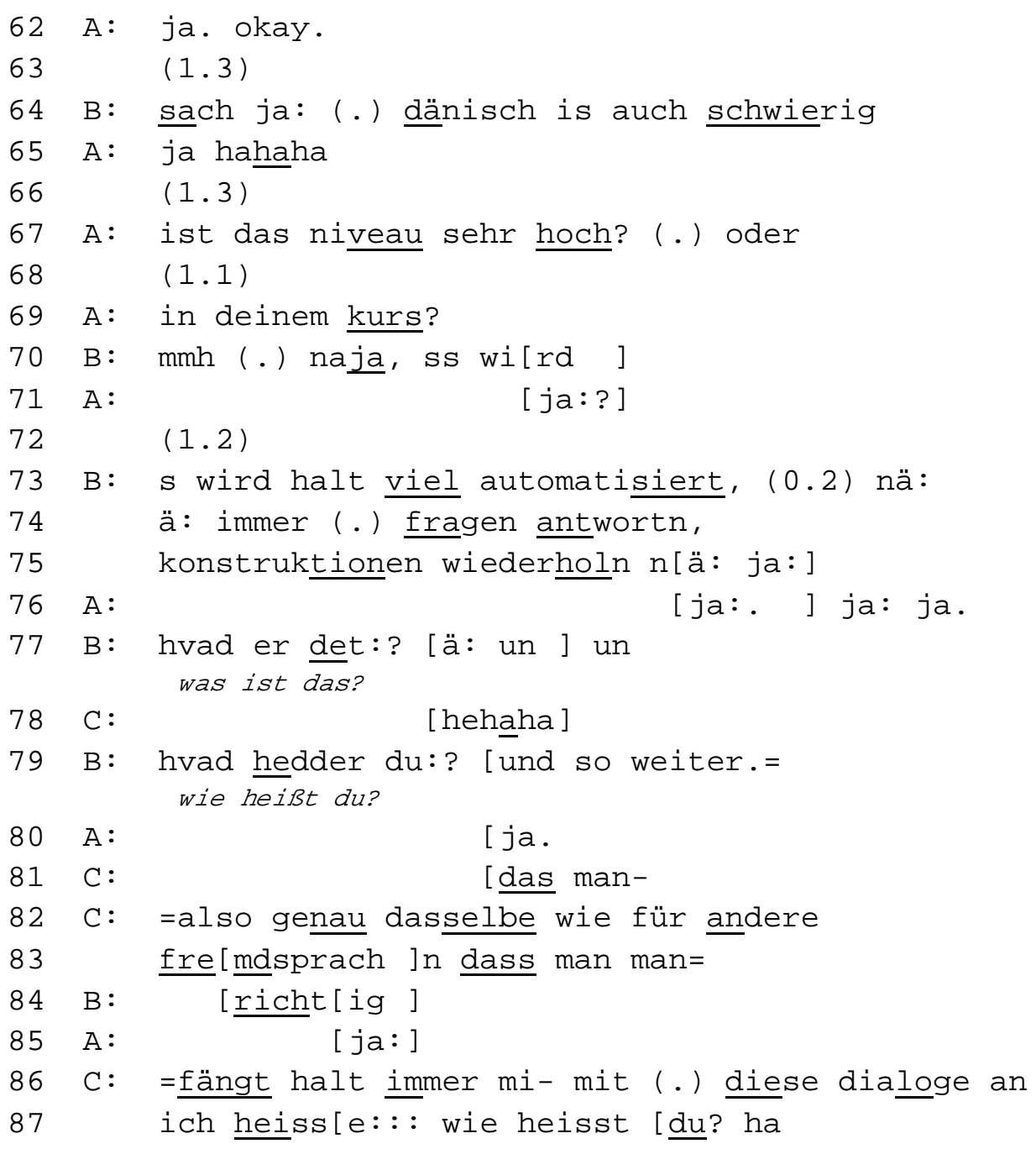




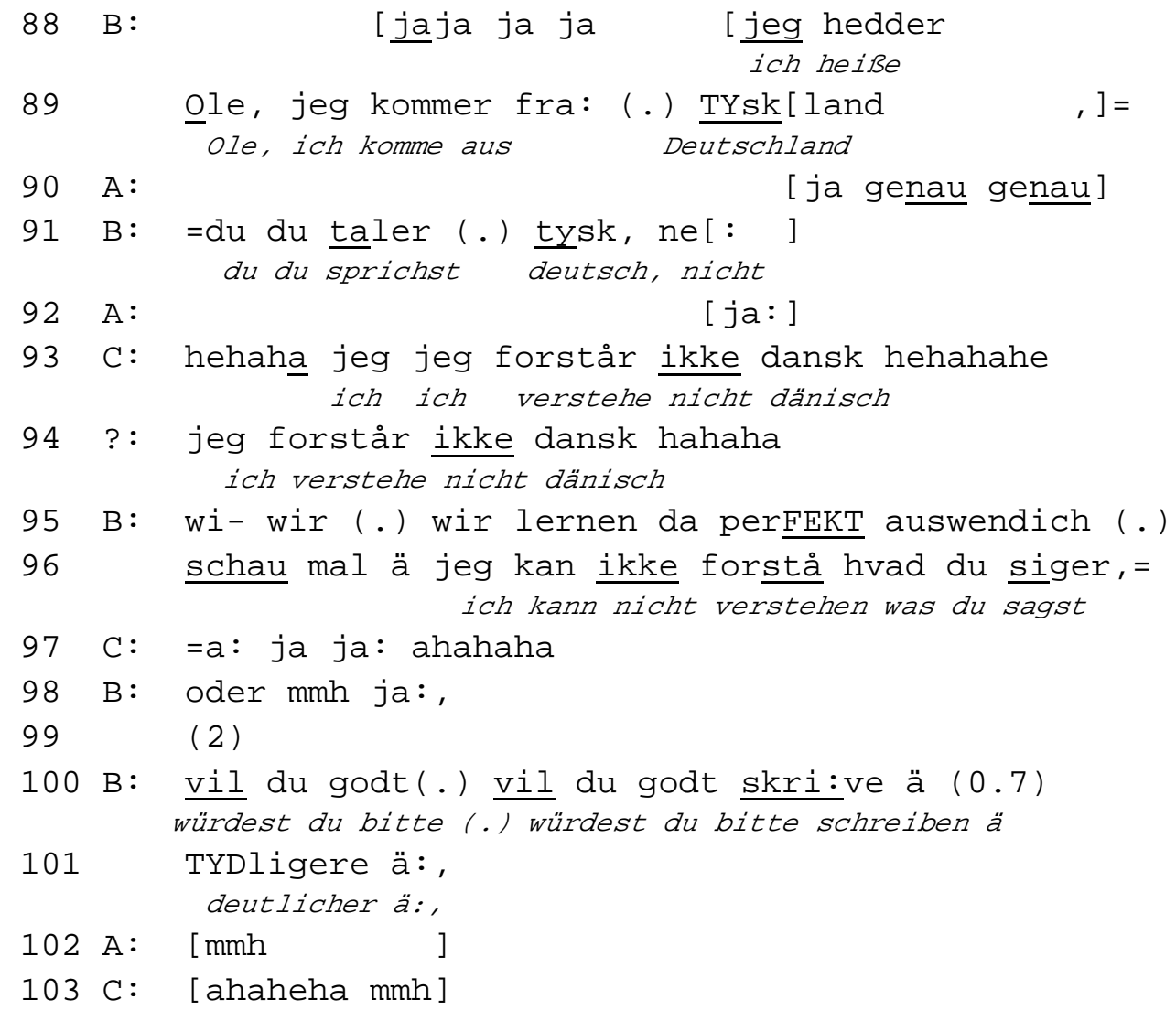

Nachdem Sprecher A in Zeile 62 wiederum Verstehen markiert hat ja. Okay, entsteht eine 1.3-sekündige Pause (63). Wie schon in den beiden Auszügen zuvor scheint eine derartig lange Pause eine Wendestelle im Gespräch zu ermöglichen und vorhersehbar zu machen. Und tatsächlich initiiert Sprecher B in Zeile 64 einen Themenwechsel, indem er sagt sach ja (.) dänisch ist auch schwierig. Hierdurch stellt er die dänische Sprache hinsichtlich ihres Schwierigkeitsgrades auf eine Stufe mit der deutschen, die Thema im vorhergehenden Auszug war. Gleichzeitig stellt er sich selbst als Dänischlernender mit dieser Äußerung auf eine Stufe mit Sprecher A und C, die jeweils Deutschlernende sind. Hier vermag Sprecher B demnach einen bestimmten Aspekt seines eigenen und des Kontextes der vorhergehenden Äußerung relevant zu machen. Zum einen den, dass er ein Wissen über den Schwierigkeitsgrad der dänischen Sprache hat. Zum anderen macht er hiermit den Aspekt seiner sprachlichen und damit auch kulturellen Zugehörigkeit relevant. Indem er dies tut, weist er den anderen Gesprächsteilnehmern eine andere als seine eigene kulturelle Zugehörigkeit zu.

Wie verhandeln nun im Folgenden die Gesprächsteilnehmer über die von Sprecher B eingeleiteten Zuweisungen? Werden die Kategorisierungen 'dänischer Nicht-Muttersprachler' vs. 'deutscher Nicht-Muttersprachler' akzeptiert? Nachdem Sprecher A zunächst in Zeile 65 lediglich zu erkennen gibt, dass er Sprecher Bs Äußerung akzeptiert, zeigt er durch seine folgende Äußerung in Zeile 67, dass er auch die darin enthaltenen Zuweisungen akzeptiert. Er tut dies, indem er den ersten Teil einer Paarsequenz in Form einer Frage formuliert ist das niveau sehr hoch? Dies gibt Sprecher B die Möglichkeit, sich als Fremdsprachenlerner mit seinen Erfahrungen zu präsentieren (Zeile 70-79). Sprecher A markiert Einverständnis mit dieser Zuweisung, indem er lediglich mit kurzen Bemerkungen zustimmt $(76,80)$. Sprecher C eröffnet in Zeile 82 die Möglichkeit einer Änderung der kulturellen Zuweisung. Er versucht 
das Thema vom fremdsprachlichen Deutschunterricht zum Fremdsprachenunterricht generell $\mathrm{zu}$ bringen also genau dasselbe wie für andere fremdsprachn. Sowohl Sprecher B als auch A geben in 84 und 85 ihre Zustimmung und tragen nun alle drei mit gemeinsamen Beteuerungen darüber, was Fremdsprachenunterricht kennzeichnet, zum weiteren Verlauf des Gesprächs bei. Hiermit weisen sich alle drei Sprecher die Kategorie 'Fremdsprachenlerner' zu.

\section{Interaktivität, kontextuelle Zuweisungen und interkulturelle Kommunikation oder wie interkulturell ist ein interkulturelles Gespräch?}

Die obenstehenden Analysen zeigen, dass Gesprächsteilnehmer schon im Laufe eines kurzen Gesprächsausschnittes wiederholt unterschiedliche kontextuelle Zuweisungen initiieren und mit ihren Gesprächsteilnehmern deren Relevanz und Gültigkeit interaktiv aushandeln. Kontextuelle Zuweisungen tragen somit zur Orientierung der Gesprächsteilnehmer darüber bei, wie und in welchem Kontext eine bestimmte Äußerung zu verstehen ist. Zusätzlich scheinen kontextuelle Zuweisungen eine wichtige Rolle bei der Themenprogression in Gesprächen zu spielen. In allen drei Auszügen ist die Initiierung einer neuen kontextuellen Zuweisung mit der Initiierung eines Themenwechsels verbunden. Die Relevanz und Gültigkeit kontextueller Zuweisungen wird von den Gesprächsteilnehmern lokal ausgehandelt, d.h. dort, wo sie initiiert werden und in direkter Relation zu dem sie vorhergehenden Kontext. Kontextuelle Zuweisungen können akzeptiert werden, sie können verändert oder aber auch zurückgewiesen werden. Sie werden aber in jedem Gespräch zu jedem Zeitpunkt zur Orientierung der Gesprächsteilnehmer von den Gesprächsteilnehmern eingesetzt. Welcher Aspekt des Kontextes im Gespräch zu welchem Zeitpunkt relevant gemacht wird, ist abhängig von den einzelnen Gesprächsteilnehmern und nicht im Vorhinein $\mathrm{zu}$ entscheiden. Auch die Tatsache, wie lange ein bestimmer Aspekt des Kontextes im Gespräch als relevant erhalten wird, ist eine interaktive Leistung der Gesprächsteilnehmer selbst. Kontext ist somit eine dynamische Größe, die in jedem Gespräch von den Gesprächsteilnehmern wiederholt eingebracht und revidiert wird und die in jedem Gespräch durch die Gesprächsteilnehmer wiederum lokal geprägt wird.

Die vorgenommenen Analysen stammen aus einem Gespräch, das als interkulturell zu bezeichnen ist: Vertreter zweier unterschiedlicher Nationen kommunizieren miteinander in einer Sprache, die für einen oder mehrere der Gesprächsteilnehmer "nicht eine Varietät seiner eigenen ist" (Knapp/Knapp-Potthoff 1990:66). Indem wir ein Gespräch als ein interkulturelles bezeichnen, wählen wir somit einen bestimmten Aspekt des Kontextes, in diesem Falle den der kulturellen Zugehörigkeit, als den das Gespräch bestimmenden Faktor aus. Interkulturell kann in diesem Zusammenhang Unterschiedliches bedeuten: dass einige Gesprächsteilnehmer nicht in ihrer Muttersprache kommunizieren und folglich mangelnde fremdsprachliche Kompetenz Ursache für Missverständnisse und Probleme ist. Es kann aber auch bedeuten, dass kulturell bedingte Unterschiede, die nicht sprachlicher Natur sind, das Gespräch auf eine bestimmte Weise prägen, die in intrakulturellen Gesprächen nicht auftritt. Gemeinsam ist diesen beiden Bedeutungen, dass Interkulturalität als der Kontextfaktor angesehen wird, der das Gespräch als solches kennzeichnet. 
Für den analysierten Gesprächsauszug kann aber nicht nachgewiesen werden, dass sich die Gesprächsteilnehmer an diesem Gespräch als an einem hauptsächlich interkulturellen orientieren. Ein Kontextfaktor wie fremdsprachliche Kompetenz, der eine Orientierung an Interkulturalität deutlich machen könnte, wird zwar thematisiert, doch er ist nicht der einzige Kontextfaktor, den die Gesprächsteilnehmer einander relevant machen. Stattdessen lässt sich eine permanente Aushandlung zwischen den Gesprächsteilnehmern über den aktuellen relevanten Kontext nachweisen. Nicht alle Äußerungen sind vor dem Hintergrund der Interkulturalität des Gesprächs angemessen zu verstehen. In einigen Fällen sind es andere Aspekte des Kontextes, die zur angemessenen Interpretation der Äußerungen in das Gespräch hereingebracht werden. In diesem Zusammenhang ist es wichtig die Bedeutung von Teilnehmerkategorien zu berücksichtigen: Die Gesprächsteilnehmer haben zu jedem Zeitpunkt des Gesprächs die Wahl, welche Teilnehmerkategorie in Bezug auf sich oder in Bezug auf einen oder mehrere der Gesprächsteilnehmer für das Verständnis ihrer Äußerung relevant ist. Hier können sie in Bezug auf das ausgewählte Gesprächssegement die Kategorie 'Inländer-Ausländer' relevant machen, sie können aber auch die Kategorie 'LernenderLehrender', die Kategorie 'Fremdsprachenlerner' oder - wie in diesem Auszug nicht geschehen, wie aber denkbar - die Kategorie 'Mann - Frau' in das Gespräch einführen.

Kann der oben analysierte Gesprächsauszug somit nicht als interkulturell bezeichnet werden? Hier gilt es zu differenzieren: Bezeichnet man den obigen Gesprächsausschnitt als interkulturell, muss man sich darüber im Klaren sein, dass die Bezeichnung interkulturell in diesem Fall eine textexterne, statische Größe ist. Sie kann Aufschluss über bestimmte Aspekte der Kommunikation geben, wie beispielsweise, dass hier Kommunikation in einer Fremdsprache stattfindet, dass es sich um Kommunikation über nationale Grenzen hinweg handelt oder dass kulturell bedingte Missverständnisse und Probleme die Kommunikation prägen. In diesem Sinne kann eine solche Bezeichnung Vorhersagen über einen bestimmten Verlauf eines solchen Gesprächs ermöglichen. Eine solche Bezeichnung sagt jedoch nicht notwendigerweise etwas darüber aus, ob sich auch die Gesprächsteilnehmer selbst an einem solchen Gespräch als interkulturell orientieren. Dies muss wiederum in jedem Einzelfall durch Mikroanalysen der Interaktion geklärt werden. Hier können beispielsweise Studien in Bezug auf Reparaturen in native-nonnative Interaktion Aufschluss darüber geben, was interkulturelle Gespräche im Einzelnen kennzeichnet (Brouwer et al. (forthc), Egbert (2002)).

Will man die Bezeichnung interkulturell als textinterne, dynamische Größe verstanden wissen, muss man demnach eine Orientierung der Gesprächsteilnehmer an diesem Kontextfaktor im Gespräch selbst nachweisen. Da nicht alle interkulturellen Gespräche von den Gesprächsteilnehmern selbst als interkulturell gestaltet werden, kann eine alleinige Fokussierung auf den Aspekt der Interkulturalität problematisch sein. So kann der Blick verbaut werden auf andere relevante Aspekte des Kontextes. Der Aspekt der Interkulturalität kann in Phasen des Gesprächs von den Gesprächsteilnehmern relevant gemacht werden, doch er ist nicht der einzige. Gesprächsteilnehmer setzen im Gespräch systematisch unterschiedliche Kontextualisierungshinweise ein, um einander gegenseitiges Verständnis zu sichern. Versteht man interkulturell demnach als eine textinterne, dynamische Größe, wird man der Tatsache gerecht, dass Kontextfaktoren nicht a priori einen gleichbleibenden, quasi 
statischen Einfluss auf das Gespräch haben. Stattdessen ermöglicht man einen Zugang zur Analyse interkultureller Kommunikation, bei dem die Gesprächsteilnehmer sich selbst und damit auch dem Analytiker zeigen, was diese Form der Interaktion zu einer interkulturellen macht.

Für das Lernziel interkulturelle Kompetenz sind Untersuchungen wie die vorliegende insofern von grundlegender Bedeutung, als sie in Frage stellen, inwieweit interkulturelle Kompetenz als ein Garant für das Gelingen interkultureller Kommunikation anzusehen ist. Gesprächsteilnehmer scheinen sich in Gesprächen, die als interkulturell bezeichnet werden können, nicht zwingend in dem Maße wie bislang angenommen an der Interkulturalität der Gesprächssituation zu orientieren. Dies bedeutet, dass ein Fokus des Lerners auf eben diesen Faktor nicht dem entspricht, was tatsächlich das Gespräch kennzeichnet. Ferner weisen Untersuchungen dieser Art darauf hin, dass eine Kommunikationssituation, die als interkulturell bezeichnet werden kann, in ihrer interaktiven Struktur nicht notwendigerweise grundlegend verschieden ist von intrakultureller. In beiden Formen der Kommunikation steht die interaktive Aushandlung von Kontextualisierungen mit dem Ziel der gegenseitigen Verständnissicherung im Zentrum. Dies stellt die Relevanz des Lernziels interkulturelle Kompetenz in Frage. Eine Alternative wäre in dem Lernziel interaktive Kompetenz zu sehen. Interaktive Kompetenz versetzt Personen in die Lage, sowohl in inter- als auch in intrakulturellen Kommunikationssituationen kompetent zu agieren. Insofern stellt sich die Frage, ob nicht die Vermittlung grundlegender interaktiver Strategien eine Alternative oder aber zumindest eine notwendige Ergänzung zu dem Lernziel interkulturelle Kompetenz sein sollte.

Um die hier angeschnittenen Fragen umfassender beantworten zu können, bedarf es weiterer sequenzieller Mikroanalysen von Gesprächssituationen, die als interkulturell bezeichnet werden können. Detaillierte Analysen authentischer Kommunikationssituationen helfen aufzuzeigen, was interkulturelle Kommunikationssituationen kennzeichnet, und sie können dazu beitragen, das homogene Bild interkultureller Kommunikation weiter zu differenzieren.

\section{Transkriptionskonventionen}

(nach Jefferson in Atkinson/Heritage (eds.) 1984: IX-XVI)

$\begin{array}{ll}\text { gut } & \text { betont } \\ \text { JA } & \text { laut } \\ \text { u : } & \text { Silbenlängung } \\ \text { gera- } & \text { abrupter Abbruch } \\ <> & \text { Tempo langsamer } \\ >< & \text { Tempo schneller } \\ \cdot & \text { fallende Intonation } \\ \text {, } & \text { fortsetzende Intonation } \\ ? & \text { steigende Intonation } \\ = & \text { direkter Anschluss zwischen zwei Äußerungen } \\ (.) & \text { Mikropause (weniger als 0.2 Sekunden) } \\ (0.5) & \text { Länge der Pause in Zehntelsekunden } \\ {[\text { ja }]} & \text { die innerhalb der Klammern stehenden }\end{array}$




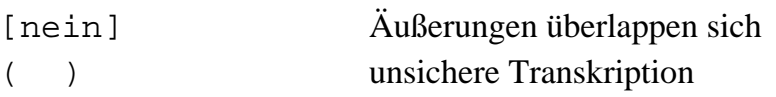

\section{Literaturangaben}

Andersen, Anne-Marie (1997): Interkulturelle Wirtschaftskommunikation in Europa: Deutschland - Dänemark. Zur Konzeption eines interkulturellen Kommunikationstrainings für den deutsch-dänischen Handel. Tostedt.

Atkinson, J. Maxwell/Heritage, John (eds.) (1984): Structures of Social Action. Studies in Conversation Analysis. Cambridge.

Bachmann, Saskia/Gerhold, Sebastian/Müller, Bernd-Dietrich/Wessling, Gerd (1995): Sichtwechsel. München.

Bergemann, Niels/Sourisseaux, Andreas L. J. (eds.) (1996): Interkulturelles Management. Heidelberg.

Brouwer, Rineke/Rasmussen, Gitte/Wagner, Johannes (forthc.): "Correcting in Seconds". In: Gardner, R./Wagner, J. (eds.): Second Language Talk. New York/London.

Egbert, Maria (2002): Der Reparatur-Mechanismus in deutschen und interkulturellen Gesprächen. Habilitation. Universität Oldenburg.

Fredsted, Elin (1998): Analyser af dansk og tysk talesprog. Oslo.

Gumperz, John J. (1982): Discourse Strategies. Cambridge.

Heritage, John (1984): Garfinkel and Ethnomethodology. Cambridge.

Hester, Stephen/Eglin, Peter (eds.) (1997): Culture in Action: Studies in Membership Categorization Analysis. Washington, D.C.

Hinnenkamp, Volker (1994): "Interkulturelle Kommunikation - strange attractions". Zeitschrift für Literaturwissenschaft und Linguistik 93: 46-74.

Kesselheim, Wolfgang (1998): "Interaktive Verfahren der Herstellung von Gruppen im Gespräch - Freiheit und Grenzen". In: Heinemann, M. (ed.): Sprachliche und soziale Stereotype. Frankfurt a.M.

Knapp, Karlfried/Knapp-Potthoff, Annelie (1990): "Interkulturelle Kommunikation". Zeitschrift für Fremdsprachenforschung 1: 62-93.

Knapp-Potthoff, Annelie (1997): "Interkulturelle Kommunikationsfähigkeit als Lernziel". In: Knapp-Potthoff, A./Liedke, M. (eds.): Aspekte interkultureller Kommunikationsfähigkeit. München: 181-205.

Koch, Wolfgang/Rossenbeck, Wolfgang (1997): "Sprachliche und soziokulturelle Probleme deutsch-schwedischer und schwedisch-deutscher Kommunikation". In: Opitz, S. (ed.): Interkulturelle Kompetenz Skandinavien - Deutschland. Ein Handbuch für Fach- und Führungskräfte. Düsseldorf: 57-90.

Krumm, Hans-Jürgen (1995): "Interkulturelles Lernen und interkulturelle Kommunikation". In: Bausch, K.-R./Christ, H./Krumm, H.-J. (eds.): Handbuch Fremdsprachenunterricht. Tübingen/Basel: 156-161.

Opitz, Stephan (1997): "Vorbemerkung: Interkulturelle Kompetenz SkandinavienDeutschland". In: Opitz, S. (ed.): Interkulturelle Kompetenz Skandinavien - Deutschland. Ein Handbuch für Fach- und Führungskräfte. Düsseldorf: 7-9. 
Rost-Roth, Martina (1994): "Verständigungsprobleme in der interkulturellen Kommunikation". Zeitschrift für Literaturwissenschaft und Linguistik 93: 9-45.

Sacks, Harvey (1992): Lectures on Conversation. Ed. by Gail Jefferson. Vol. I, II. Oxford.

Sacks, Harvey/Schegloff, Emanuel A./Jefferson, Gail (1974): "A simplest systematics for the organization of turn-taking for conversation". Language 50/4: 696-735.

Schegloff, Emanuel A. (1982): "Discourse as an interactional achievement: Some uses of 'uh huh' and other things that come between sentences". In: Tannen, D. (ed.): Analyzing Discourse: Text and Talk. Washington D.C.: 71- 93.

Schiffrin, Deborah (1994): Approaches to Discourse. Oxford. 